Vol. 16, Núm. 2

Abr.-Jun. 2020. pp 84-95 doi: $10.35366 / 94538$

https://dx.doi.org/10.35366/94538

\title{
Medidas, recomendaciones y factores de riesgo en cirugía de mano en tiempo de COVID-19
}

\author{
Measures, recommendations and risk factors in hand surgery in times of COVID-19

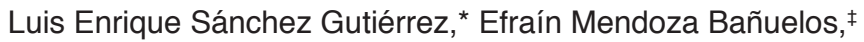 \\ José Alberto López González ${ }^{\ddagger}$
* Médico adscrito de Cirugía de Mano y Microcirugía.
${ }^{\ddagger}$ Residente de Cirugía de Mano y Microcirugía. \\ Hospital Regional de Alta Especialidad Regional del Bajío. \\ Correspondencia: Dr. Luis Enrique Sánchez Gutiérrez. Blvd. Milenio No. 130, \\ Col. San Carlos la Roncha, 37660, León, Guanajuato. Correo electrónico: frainxootbaak@outlook.com
}

\section{RESUMEN}

La nueva pandemia de coronavirus, también conocida como SARS-CoV-2, ha ejercido una inmensa presión sobre los sistemas de salud en todo el mundo. En consecuencia, múltiples gobiernos federales y estatales han impuesto restricciones a los hospitales, como la limitación de la "cirugía electiva" y han recomendado el distanciamiento social o físico. Revisamos la literatura en áreas que han sido afectadas, incluyendo la selección quirúrgica y la atención a los pacientes en cirugía de mano. En estas áreas se han visto afectados paradigmas respecto a pacientes hospitalizados y ambulatorios, incluyendo la prioridad quirúrgica, la prioridad física y el distanciamiento social, priorizándose los factores de riesgo. Durante esta crisis es importante que la cirugía de mano y microcirugía hagan hincapié en la seguridad del personal y en la ralentización de la propagación del virus para poder seguir manteniendo la vitalidad de sus funciones. Esta revisión de la literatura trata de proporcionar una orientación basada en pautas de prevención, comprender sobre cirugía electiva, de urgencia y participar en rutinas

\section{ABSTRACT}

The new coronavirus pandemic, also known as SARS-CoV-2, has put immense pressure on health systems around the world. As a result, multiple federal and state governments have imposed restrictions on hospitals, such as limiting "elective surgery" and recommending social or physical distancing. We reviewed the literature in areas that have been affected, including surgical selection, patient care in hand surgery. These areas have been affected in inpatient and outpatient paradigms including surgical priority, physical priority, or social distancing priority for risk factors. During this crisis, it is important that hand surgery and microsurgery emphasize staff safety and slowing the spread of the virus in order to maintain the vitality of their functions. This review of the literature aims to provide guidance based on prevention guidelines, to understand elective surgery also in emergencies and to participate in routines that improve the well-being of the physician, as well 
que mejoren el bienestar del médico, así como detectar oportunamente factores de riesgo que sean predictivos a riesgo de contagio de infección.

Palabras clave: COVID-19, factores de riesgo, cirugía, electiva, hospitalización, cirugía de mano. as to detect early risk factors that are predictive of risk of infection.

Keywords: COVID-19, risk factors, surgery, elective, hospitalization, hand surgery.

A medida que la pandemia de la enfermedad coronavirus 2019 (COVID-19) comience a aflojar su curva inicial en el mundo y contemplemos el inicio del camino de vuelta a la «normalidad», la comunidad médica se enfrentará a muchos desafíos, pero ninguno más importante que evitar la propagación de COVID-19 y limitar la posibilidad y el alcance de una posible «segunda ola».

El propósito del presente informe es proporcionar una lista de factores de riesgo y recomendaciones encaminadas a reducir la transferencia de patógenos durante la reintroducción de procedimientos quirúrgicos ortopédicos electivos (cirugía de la mano), centrándose específicamente en la prevención de la propagación del síndrome respiratorio agudo severo (SRAS)-infección por el virus CoV-2.

Aunque suponemos que operaremos a pacientes sin infección por SARSCoV-2, debido a las complejidades que entraña el diagnóstico preciso de la infección por SARS-CoV-2, incluidos hasta $40 \%$ de resultados falsos negativos en las pruebas de reacción en cadena de la polimerasa de transcripción inversa (RT-PCR), ${ }^{1}$ se considera como necesario tomar precauciones para reducir al mínimo la posibilidad de transmisión de la infección por parte de los pacientes potencialmente infectados. ${ }^{1-3}$

Somos conscientes de que la situación evoluciona a diario y que algunas de las recomendaciones del presente informe tal vez deban modificarse a medida que surjan nuevas pruebas. Además, las medidas de prevención de infecciones descritas, así como los factores de riesgo dependerán en gran medida de la prevalencia de COVID-19 en las zonas afectadas y de la capacidad de aplicar las pruebas diagnósticas recomendadas para descartar adecuadamente la presencia de COVID-19 antes de la cirugía.

El día 26 de marzo de 2020 se publicó en la página oficial del colegio real de Inglaterra una guía de práctica clínica con orientación sobre la práctica para cirujanos que trabajan durante la pandemia de COVID-19, anunciándolo de esta forma:

1. Introducción.

1.1 El personal quirúrgico deberá adaptarse durante la pandemia de COVID-19.

1.2 Las prioridades en la lista de importancia son:

I. Mantener las capacidades de cirugía de emergencia.

II. Proteger y preservar al personal quirúrgico.

III. Cumplir funciones quirúrgicas alternativas.

IV. Cumplir funciones no quirúrgicas alternativas. 
1.3 Es probable que el personal quirúrgico se agote a medida que los médicos se autoaíslan. El mantenimiento de los servicios de emergencia normales de la subespecialidad quirúrgica será cada vez más difícil.

1.4 Es probable que disminuya la capacidad del quirófano. Es probable que los cirujanos sean redistribuidos para apoyar a las especialidades no quirúrgicas.

1.5 Los principios generales son:

I. Triage y prestación de asistencia sanitaria a los pacientes para obtener el máximo beneficio, como en el caso de las víctimas masivas.

II. Proteger y preservar al personal quirúrgico.

1.6 Cualquier plan debe ser dinámico, reactivo y cambiará a medida que la situación/escala se desarrolle. Los cirujanos tendrán que ser flexibles, colaborar y mostrar liderazgo en lo que se espera que sean tiempos de prueba.

2. Prioridad 1: Mantener las capacidades de cirugía de emergencia.

2.1 El objetivo principal del personal quirúrgico es mantener la prestación de servicios de cirugía de emergencia, incluidos los traumatismos graves.

2.2 Inicialmente esto se llevará a cabo mediante rotaciones individuales de especialidad. Se incluirán rotaciones en las que algunos miembros del equipo no entran a trabajar y actúan como una reserva saludable.

2.3 Si la fuerza de trabajo se reduce debido a una enfermedad, es posible que las rotaciones individuales de especialidad ya no sean sostenibles. En ese caso, puede ser necesario pasar a una rotación genérica de cirujanos. Éste se basaría en las competencias y se encargaría del triage inicial, parte de la cirugía y la gestión postoperatoria.

2.4 Es probable que el personal se divida en cirujanos de tórax y cardiovasculares (vasculares, generales, urología) y cirujanos de extremidades (cirugía de mano, ortopedistas, plásticos). El cirujano genérico necesitaría el apoyo virtual de especialistas. Algunas de las operaciones seguirán requiriendo un cirujano especialista cuando esté disponible.

2.5 Se sugiere que cada sitio tenga la presencia de un cirujano de tórax y un cirujano de extremidades en grados medios. La pauta de turnos sería de 24 o 12 horas, según el número de cirujanos y el ritmo.

2.6 La vía del traumatismo grave se ha identificado como una prioridad. Es posible que se requiera que los cirujanos se encarguen de dirigir el Servicio de Urgencias médico-quirúrgicas, incluyendo el papel de líder del equipo de trauma (LET), dependiendo de las disposiciones locales.

2.7 Es posible que se requieran soluciones regionales si las unidades quirúrgicas más pequeñas colapsan. Es probable que las redes de traumatología mayor existentes sean el mejor vehículo para lograrlo.

3. Prioridad 2: Proteger y preservar al personal quirúrgico.

3.1 Es vital que se minimice el riesgo para el personal. Esto puede lograrse mediante:

I. Se deben utilizar soluciones no quirúrgicas para evitar la cirugía siempre que sea posible. 
II. Equipo de protección personal (EPP) utilizado correctamente de acuerdo con la orientación nacional.

III. El descanso y la recuperación se tienen en cuenta en toda la planificación.

IV. El apoyo psicológico se tiene en cuenta en toda la planificación. ${ }^{4}$

En resumen, COVID-19 representa un reto incierto que podría generar un gran número de pacientes en un periodo corto y mediano de tiempo. La mejor manera de manejar esto está evolucionando. No habrá una solución ideal, así que se pide trabajar en equipo para resolver el desafío.

Asimismo, en el Gobierno de México se inicia con la diferenciación de situaciones médicas, así como observar factores para valorar:

Caso sospechoso: persona de cualquier edad que en los últimos siete días haya presentado al menos dos de los siguientes signos y síntomas: tos, fiebre o cefalea*, acompañadas de al menos uno de los siguientes signos o síntomas:

- Disnea (dato de gravedad)

- Artralgias

- Mialgias

- Odinofagia/ardor faríngeo

- Rinorrea

- Conjuntivitis

- Dolor torácico

*En menores de cinco años, en lugar de dolor de cabeza (cefalea) se puede presentar irritabilidad. [Los criterios están sujetos a cambios y serán actualizados de acuerdo a la disponibilidad de información adicional]..$^{5}$

En la valoración de conceptos actuales y revisión de cirugía electiva de cirugía de mano durante la pandemia COVID-19, en la que se realiza una serie de preguntas a 77 expertos cirujanos ortopédicos, infectólogos, microbiólogos, virólogos y anestesiólogos, ${ }^{6}$ y recordando que este tipo de estudio tiene su limitación sobre el nivel de evidencia, así como diferentes puntos de grados de recomendación, se realizaron una serie de preguntas con diferentes apartados, tales como tema en general, escenarios prequirúrgicos, transquirúrgicos y postquirúrgicos.

La pregunta número 4 del cuestionario II dice: ¿qué medidas adicionales deberían adoptarse durante el proceso previo al ingreso de los pacientes que se someten a una cirugía electiva durante la pandemia de SARS-CoV-2? La respuesta/recomendación $(R, R)$ : se debe realizar y documentar el examen de los síntomas de COVID-19. Las preguntas deben tener como objetivo preguntar a los pacientes sobre los síntomas de COVID-19 (fiebre, dificultad para respirar, tos, pérdida del olfato y el gusto, diarrea, dolor de cabeza, dolor de garganta), así como información sobre el TOCC (viajes a regiones con alta prevalencia de COVID-19, ocupación con alto riesgo de infección por COVID-19, contacto con 
personas que se sabe que están infectadas por COVID-19, o cercanía con un caso positivo). Es decir, aquellas personas con alto riesgo de infección deben ser operadas y puestas en cuarentena según las directrices locales, a menos que se puedan realizar las pruebas adecuadas para descartar la presencia de COVID-19.

- Si se realiza la prueba de reacción en cadena de la polimerasa de transcriptasa inversa (RT-PCR) para el SARS-CoV-2 debe realizarse dentro de los tres a siete días de la cirugía electiva y documentarse en los cuadros clínicos del paciente. Se deben realizar procedimientos de selección clínica y/o microbiológica apropiados para cada paciente.

Otra pregunta menciona: ¿deberían los pacientes que se someten a una cirugía electiva ser sometidos a pruebas de detección del SARS-CoV-2? Si es así, ¿las pruebas deben hacerse a todos los pacientes o a un grupo selecto? $R$, R: las pruebas de RT-PCR para el SARS-CoV-2 son cada vez más accesibles. Las pruebas en los pacientes deberían ser obligatorias en las zonas de alta prevalencia, dado el riesgo de transmisión de la enfermedad por parte de los pacientes asintomáticos. Las pruebas de rutina no son factibles en todos los lugares debido a las limitaciones en la capacidad de realización de pruebas y, por consiguiente, se deberían seguir las directrices locales en estas zonas. En su justificación, se ha descrito anteriormente, los pacientes con un historial de viajes o estancias en zonas con una alta prevalencia de SARS-CoV-2 o de contacto directo con personas que han dado positivo en la prueba de COVID-19 deben ser sometidos a la prueba. Además, hay otras características clínicas que pueden indicar una posible infección por COVID-19..$^{7-10}$

\begin{tabular}{lr} 
Síntoma & $\mathrm{n}(\%)$ \\
\hline Fiebre & $83(99.0)$ \\
Tos & $59(82.0)$ \\
Fatiga & $44(70.0)$ \\
Anorexia & $40(84.0)$ \\
Disnea & $31(40.0)$ \\
Producción de esputo & $28(33.0)$ \\
Pérdida de gusto y olfato & $18.6(33.9)$ \\
Dolor de garganta & $5(17.4)$ \\
Diarrea & $2(12.5)$ \\
Congestión nasal & $4(4.80)$ \\
\hline
\end{tabular}

Un síntoma que se ha discutido cada vez más es la pérdida del olfato y el gusto, ${ }^{10}$ particularmente en ausencia de congestión nasal y rinorrea. ${ }^{9-13}$ Es probable que los principales hallazgos clínicos en el escenario de un entorno de detección sean la presencia de fiebre, falta de aliento, tos y anorexia. Se ha notificado la infección simultánea con otros patógenos respiratorios, principalmente virales, en hasta $21 \%$ de los pacientes con infección por el SARS-CoV-2, 
y debería existir un alto grado de sospecha de coinfección. ${ }^{10}$ Siendo éste un grado de recomendación alto con $96.25 \%$ de acuerdo, $1.25 \%$ en desacuerdo y $2.5 \%$ con abstención.

Otro cuestionamiento refiere: ¿debería retrasarse la cirugía electiva en pacientes geriátricos o con comorbilidades que los pongan en alto riesgo de contraer COVID-19? Este grupo opina que la cirugía electiva debe estratificarse en función del riesgo y posiblemente aplazarse en función de la edad del paciente (> 75 años), la obesidad mórbida, la diabetes, la hipertensión no controlada, la enfermedad pulmonar crónica, la apnea obstructiva del sueño, la cardiopatía crónica y el estado inmunológico comprometido (por ejemplo: los pacientes con trasplante de órganos o de médula ósea, cáncer en fase activa, los que reciben o han recibido recientemente quimioterapia o radioterapia) en los primeros días en que se reanuda la cirugía electiva. Los pacientes con comorbilidades y factores de riesgo significativos deben programarse después de que los pacientes más sanos hayan sido tratados y se haya acumulado experiencia en el establecimiento de protocolos de detección, prevención y tratamiento.

Tabla 1: Recomendaciones de los Centros de Servicios de Medicare y Medicaid sobre cirugía electiva y procedimientos para adultos; recomendaciones del Colegio Americano de Cirujanos para el manejo de procedimientos quirúrgicos electivos.

Cirugía de mano

\begin{tabular}{|c|c|c|c|}
\hline \multicolumn{2}{|c|}{ Fase II } & \multicolumn{2}{|c|}{ Fase III } \\
\hline Programar & Reprogramar & Programar & Reprogramar \\
\hline $\begin{array}{l}\text { Herida con tendón, lesión } \\
\text { nerviosa }\end{array}$ & $\begin{array}{l}\text { Herida en cicatrización sin } \\
\text { lesión de tendón, lesión } \\
\text { nerviosa }\end{array}$ & $\begin{array}{l}\text { Herida aguda con lesión } \\
\text { de tendón, lesión nerviosa } \\
\text { con dos semanas }\end{array}$ & Herida de $>2$ semanas \\
\hline Infección aguda & $\begin{array}{l}\text { Infección crónica } \\
\text { y en curación }\end{array}$ & Infección aguda & $\begin{array}{l}\text { Infección crónica, osteo- } \\
\text { mielitis }\end{array}$ \\
\hline $\begin{array}{l}\text { Fracturas agudas, mano, } \\
\text { muñeca, codo que requie- } \\
\text { re Tx quirúrgico }\end{array}$ & $\begin{array}{l}\text { Tendinitis mano, muñeca, } \\
\text { codo, dedo en gatillo, } \\
\text { tenosinovitis, epicondilitis }\end{array}$ & $\begin{array}{l}\text { Fracturas aguas que re- } \\
\text { quieren manejo quirúrgico }\end{array}$ & $\begin{array}{l}\text { Tendinitis mano, muñeca, } \\
\text { codo, tenosinovitis } \\
\text { estenosante, tenosinovitis, } \\
\text { epicondilitis }\end{array}$ \\
\hline \multirow[t]{4}{*}{$\begin{array}{l}\text { Laceración aguda en } \\
\text { mano, muñeca, codo de } \\
\text { dos semanas }\end{array}$} & $\begin{array}{l}\text { Síndromes de } \\
\text { compresión nerviosa }\end{array}$ & $\begin{array}{l}\text { Lesión aguda de alta } \\
\text { energía con dolor en } \\
\text { mano, muñeca, codo sin } \\
\text { evaluación previa }\end{array}$ & $\begin{array}{l}\text { Síndromes de compresión } \\
\text { nerviosa }\end{array}$ \\
\hline & $\begin{array}{l}\text { Fractura crónica } \\
>6 \text { semanas }\end{array}$ & & Fracturas $>2$ semanas \\
\hline & Lesión de mano, muñeca & & Lesión de mano, muñeca \\
\hline & 0 codo $>2$ semanas & & \\
\hline
\end{tabular}


Tabla 2: Urgencia estratificada de diferentes diagnósticos ortopédicos y procedimientos quirúrgicos para cirugías de pacientes hospitalizados y/o ambulatorios en cirugía de mano.

\begin{tabular}{|c|c|c|}
\hline $\begin{array}{l}\text { Prioridad A emergencia } \\
\text { (dentro de } 24 \text { horas) }\end{array}$ & $\begin{array}{l}\text { Prioridad B urgente } \\
\text { (dentro de } 48 \text { horas) }\end{array}$ & $\begin{array}{l}\text { Prioridad C expedito } \\
\text { (dentro de dos semanas) }\end{array}$ \\
\hline $\begin{array}{l}\text { Fracturas de mano con lesión } \\
\text { vascular }^{\star} \\
\text { Síndrome compartimental } \\
\text { Reducción de articulación luxada** } \\
\text { Fractura de mano cerrada con inmi- } \\
\text { nente compromiso en tejidos blandos } \\
\text { Fracturas de mano expuestas } \\
\text { Síndrome agudo del túnel del carpo } \\
\text { Tenosinovitis de flexores piógena } \\
\text { Reimplantación digital }\end{array}$ & & $\begin{array}{l}\text { Fractura quirúrgica en mano cerradas } \\
\text { sin compromiso neurológico, vascular } \\
\text { Lesiones de tendinosa, sin compro- } \\
\text { miso inminente en tejidos blandos } \\
\text { Fracturas quirúrgicas de clavícula, } \\
\text { húmero, radio, cúbito }\end{array}$ \\
\hline Prioridad D (dentro de tres meses) & Prioridad E (más de tres meses) & \\
\hline Síndrome crónico del túnel del carpo & $\begin{array}{l}\text { Tenosinovitis estenosante sin } \\
\text { compromiso neurológico, vascular }\end{array}$ & \\
\hline
\end{tabular}

Con la justificación que toda cirugía de mano electiva y de urgencia debe planificarse y organizarse con antelación para lograr resultados óptimos. Por ejemplo, en los procedimientos de artroplastia, la diabetes, la obesidad u otros factores de riesgo modificables conocidos, descritos en los criterios de la Reunión Internacional de Consenso (ICM), deberían optimizarse antes del procedimiento planificado para reducir al mínimo las complicaciones postoperatorias. ${ }^{8}$ Sin embargo, no existen sistemas de estratificación de riesgos específicos del SARS-CoV-2, basados en pruebas, para los procedimientos de cirugía de mano electivos y de urgencia. No obstante, en retrospectiva se ha tomado a consideración la toma de decisión para eventos quirúrgicos primeramente basado en los tipos de fase epidemiológica, en donde la Asociación Americana de Cirujano Ortopedistas (AAOS) apoya las recomendaciones sobre el retraso de las cirugías electivas defendidas por los Centros de Servicios de Medicare y Medicaid (CMS), el Colegio Americano de Cirujanos (ACS) y el Cirujano General de los Estados Unidos. ${ }^{12}$ (Tabla 1).

Asimismo, Massey y colaboradores realizan una revisión sobre valoración, dependiendo por prioridades urgencia y emergencia a resolver en 24 a 48 horas, así como estadificación de prioridades y tiempo de resolución, que se publicará en junio de 2020; además, sugieren la estrategia con base en prioridades y en recursos necesarios para priorizar diferentes cirugías y el traslado a diferentes unidades ambulatorias para mitigar el riesgo y conservar los recursos en el hospital del paciente. Las cirugías que pueden ser pospuestas en diferentes fases de esta pandemia ${ }^{13}$ (Tabla 2). 
Varios estudios han analizado los factores de riesgo del paciente que pueden estar asociados con un curso clínico más grave de la infección por COVID-19. Se ha considerado que varios grupos de pacientes son extremadamente vulnerables a enfermedades importantes y potencialmente mortales si se exponen al SARS-CoV-2. ${ }^{5}$

Grupos de riesgos basados en guía de práctica clínica del Gobierno de México

Grupos de riesgo 1:

- Menores de cinco años, especialmente $<2$ años.

- Personas > 65 años.

- Enfermedad pulmonar como EPOC, fibrosis quística y asma.

- Enfermedad cardiovascular, excepto hipertensión arterial aislada.

- Nefropatía.

- Hepatopatía.

Grupos de riesgo 2:

- Enfermedad hematológica, incluyendo anemia falciforme.

- Alteraciones metabólicas como diabetes mellitus tipo 1,2 y gestacional, incluso bajo control.

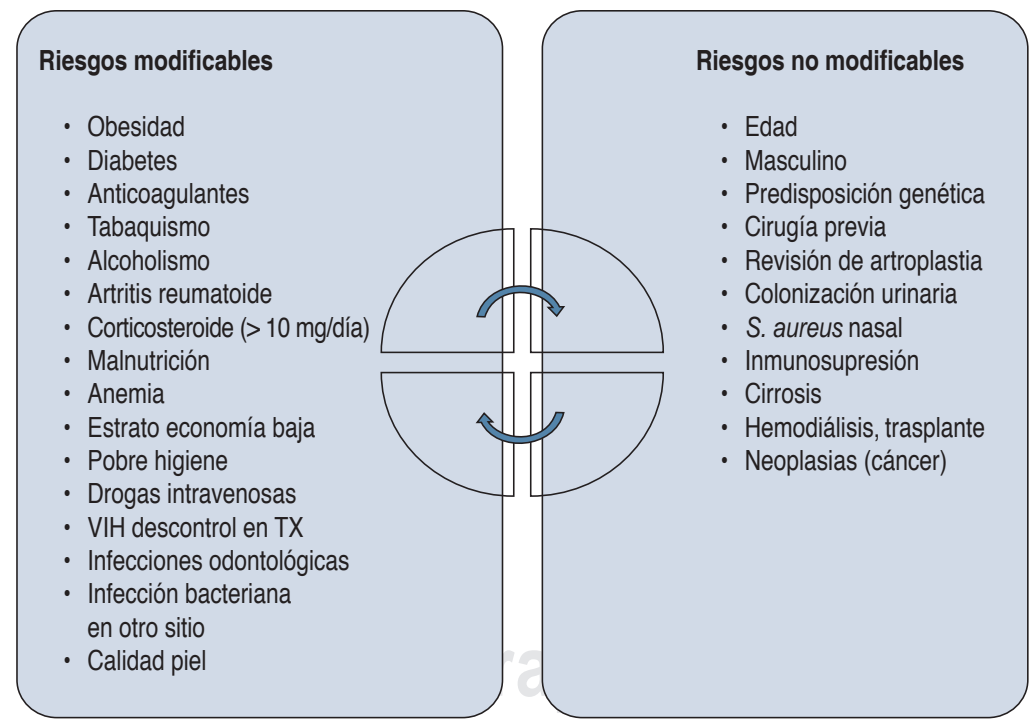

Figura 1: Esta imagen ejemplifica los factores de riesgo modificables y no en cirugías ortopédicas. Basados en: Courtney PM, Whitaker CM, Gutsche JT, Hume EL, Lee GC. Predictors of the need for critical care after total joint arthroplasty: an update of our institutional risk stratification model. Journal of Arthroplasty. 2014; 29 (7): 1350-1354. Available in: https://www.ncbi.nlm.nih.gov/pubmed/. 


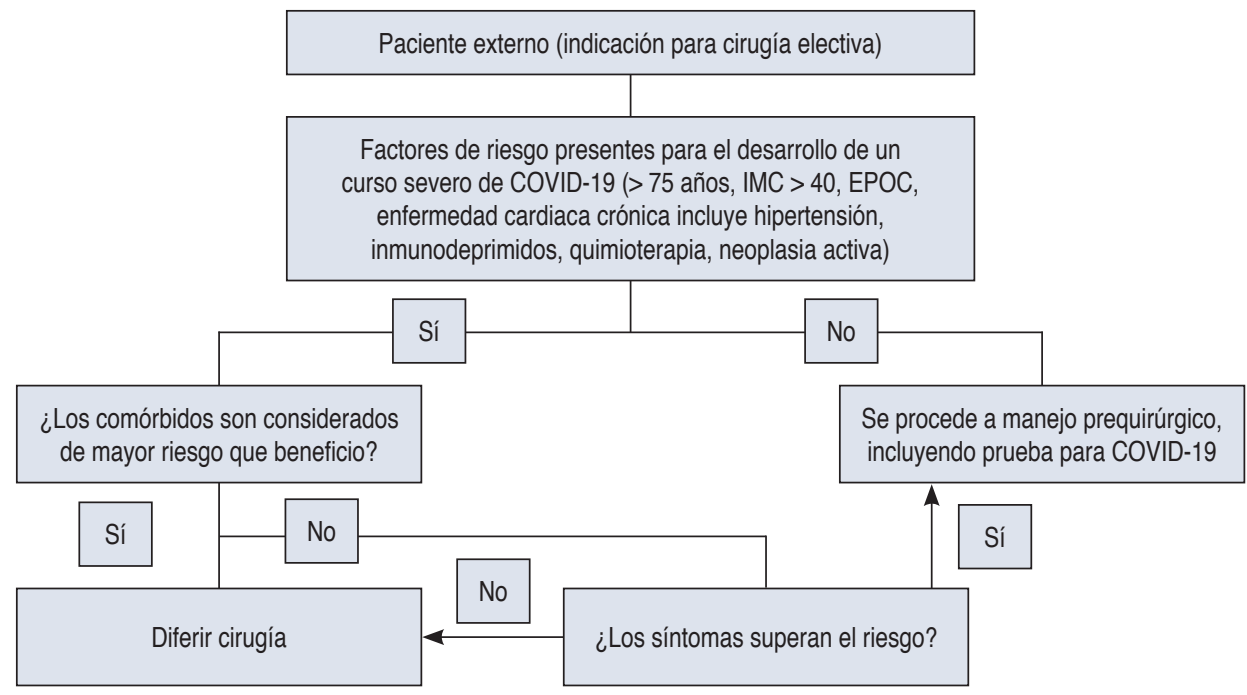

Figura 2: Algoritmo para el manejo de la cirugía de mano. Basado en: Parvizi J y colaboradores. Characteristics and early prognosis of COVID-19 infection in fracture patients. J Bone Jt Surg. 2020: 1

Mi B, Chen L, Xiong Y, Xue H, Zhou W, Liu G. Characteristics and early prognosis of COVID-19 infection in fracture patients. J Bone Joint Surg Am. 2020; 102 (9): 750-758.

Publicado por: Chen L, Xiong colaboradores. Cita en resuming elective orthopaedic surgery during the COVID-19 pandemic: guidelines developed by the international consensus group.

- Afección neurológica (cerebral, espinal, nervios periféricos, parálisis, síndromes epilépticos, evento vascular cerebral y afección intelectual) o del neurodesarrollo.

- Inmunosupresión inducida por medicamentos.

Grupos de riesgo 3:

- Personas que viven con VIH.

- Personas que viven en asilos u otros centros de cuidados a largo plazo.

- Mujeres embarazadas y hasta dos semanas de puerperio.

- Personas que tienen obesidad, con un índice de masa corporal (IMC) $\geq 40$ $\mathrm{kg} / \mathrm{m}^{2}$.

Además de la edad avanzada se han evaluado varios factores de riesgo en la pandemia actual, de los cuales se han identificado algunas comorbilidades (como la hipertensión) como los principales factores de riesgo de enfermedad grave y muerte; sin embargo, en análisis multivariado de pacientes hospitalizados con COVID-19 grave, ninguna comorbilidad estuvo significativamente asociada al resultado, es decir, si se suman el número de comorbilidades en un solo individuo son las que pueden incrementar la tasa de mortalidad y el riesgo de caso grave ${ }^{14,15}$ (Figura 1). 
Citando a Lipira y colaboradores, ${ }^{16}$ en su estudio de complicaciones a los 30 días de la cirugía de la mano: se ha utilizado anteriormente un análisis de 10,646 pacientes en un periodo que va de 2006 a 2011, para cuantificar las complicaciones e identificar los factores de riesgo en un intento de determinar la incidencia de las complicaciones postoperatorias, después de cirugía de la mano e identificar los factores asociados con el aumento del riesgo de complicaciones, a fin de centrar las estrategias preventivas. Fueron analizados por su asociación con complicaciones en el periodo postoperatorio de 30 días, usando tanto los análisis univariantes como los multivariantes, con resultados $2.5 \%$ de incidencia general de complicaciones en los 30 días posteriores de la cirugía de la mano. (Intervalo de confianza de 95\%, 2.2 a 2.8\%). En el que el análisis de univariable edad avanzada, diabetes, enfermedad pulmonar obstructiva crónica, insuficiencia cardiaca congestiva, aterosclerosis, esteroides, trastorno hemorrágico, aumento de la tasa de mortalidad y aumento de la clase de heridas, procedimiento de emergencia, más largo el tiempo operativo y la transfusión preoperatoria se asociaron con un riesgo significativamente mayor de complicaciones, y la anestesia local y la cirugía ambulatoria se asociaron con un menor riesgo. En el modelo multivariante, sexo masculino y la transfusión preoperatoria se asociaron con un riesgo significativamente mayor, y la cirugía ambulatoria se asoció con un riesgo significativamente menor. La complicación más común fue la infección del sitio quirúrgico (1.2\%) y por conclusión la incidencia de las complicaciones fue baja, y el estado general de salud fue más importante que las comorbilidades específicas en la predicción del riesgo de complicaciones. Esta información puede ser valiosa para asesorar a los pacientes antes de la cirugía y para identificar a los pacientes con mayor riesgo de complicaciones después de la cirugía de la mano, con nivel de evidencia III. ${ }^{16}$

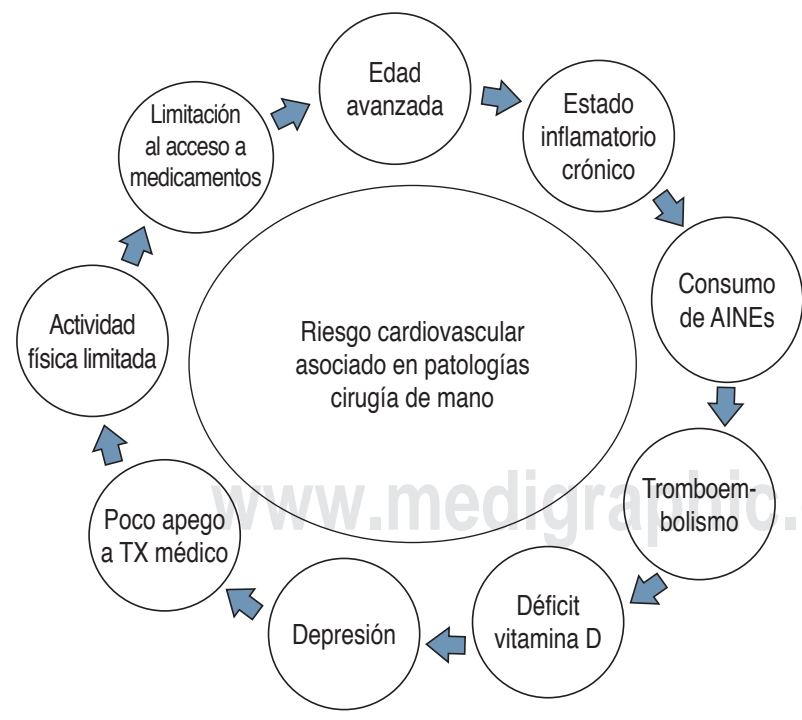

Figura 3:

Vínculo de enfermedades ortopédicas y cardiovasculares. Tomado de: Bhattacharyya T, lorio R, Healy WL. Rate of and risk factors for acute inpatient mortality after orthopaedic surgery. J Bone Joint Surg Am. 2002; 84 (4): 562-572. Available in: https:// www.ncbi.nlm.nih.gov/pubmed/. 
Por análisis, discusión y conclusiones: en cirugía de mano y microcirugía requieren protocolos de seguridad cuando se atiende a pacientes con riesgo de infección o transmisión de COVID-19 y comorbilidades en el entorno hospitalario ambulatorio y hospitalario. Asimismo el conocimiento reciente de la capacidad capsular de este nuevo virus, de adherirse a superficies metálicas y plásticas y el reto de la búsqueda de un tratamiento incrementan los retos de la pandemia que enfrentamos. ${ }^{13}$ Hicimos un análisis de sus consecuencias en la población de pacientes más frágil. Al determinar la necesidad de medidas preventivas especiales como la limpieza de superficies, bastones, andadores y muletas después de estar en un área interior o exterior poblada y para pacientes postoperatorios con cirugía de mano y microcirugía en dispositivos quirúrgicos, puede estar presente una biopelícula viral y comorbilidades en un entorno ambulatorio que recibe inhibidores de la ECA y terapia antiinflamatoria, los médicos ortopédicos deben cambiar el plan farmacológico y educar en nuevas medidas preventivas sobre el uso de dispositivos ortopédicos. Se necesitan nuevas líneas de investigación. ${ }^{17-20}$ Sin embargo, las comorbilidades que ponen a las personas en mayor riesgo en el análisis de esta pandemia relacionado con factor de riesgo de cirugía de mano son:

- Hipertensión.

- Enfermedad cardiaca/enfermedad cerebrovascular.

- Diabetes.

- Enfermedad respiratoria.

- Malignidad e inmunodeficiencia.

- Las personas tratadas con inhibidores de la ECA y Bloqueador de ReninaAngiotensina tienen mayor riesgo.

- EPOC es la comorbilidad más predictiva tanto para la enfermedad grave como para el ingreso en la UCl.

- Patología ortopédica que representa factor de riesgo.

- Enfermedades reumáticas que generen inmunodeficiencia celular.

- Fractura infectada/no unión infectada.

- Tumor ortopédico con alto grado de agresividad/malignidad.

- Evento quirúrgico que requiera tratamiento quirúrgico más dos horas.

- Osteomielitis.

Por consiguiente, se toma a consideración para el manejo de cirugía de la mano el siguiente algoritmo (Figura 2) para información de paciente, familiar y metodología en toma de decisiones.

Asimismo, en el pasado diagrama de flujo adaptado lleva al análisis que patológicas ortopédicas y traumáticas en cirugía de mano que incluyan: limitación de actividad, que genere inmovilización, desnutrición y estadios que tornen al paciente a inmunosupresión. Estos serán tomados como factores de riesgo, así como la oportuna detección de procesos patológicos que integran un círculo de estados comórbidos que pueden llegar a empeorar el pronóstico, en particular del paciente (Figura 3), por lo cual cabe señalar tomar en cuenta dichas recomendaciones que van actualizándose cada día y verificar su peso en la evidencia de la práctica. 


\section{BIBLIOGRAFÍA}

1. American Hospital Association. Safely resuming elective surgery as COVID-19 curve flattens: ACS, ASA, AORN and AHAdevelop roadmap for readiness. 2020 Apr 17. [Accessed 2020 Apr 22].

2. UpToDate. Patient education: coronavirus disease 2019 (COVID-19) overview (the basics). [Accessed 2020 Apr 21] Available in: https://www.uptodate.com/contents/coronavirus-disease-2019-covid-19overview-the-basics?topicRef $=127551$ \&source=see_link.

3. Testing for COVID-19 I CDC. [Accessed April 21, 2020] Available in: https://www.cdc.gov/ coronavirus/2019-ncov/symptoms-testing/testing.html..

4. https://www.rcseng.ac.uk/coronavirus/joint-guidance-for-surgeons-v1

5. Lineamiento estandarizado para la vigilancia epidemiológica y por laboratorio de COVID-19. Disponible en: https://www.gob.mx/salud/documentos/nuevo-coronavirus

6. Parvizi J, Gehrke T, Krueger CA, Chisari E, Citak M, Van OS. Resuming elective orthopaedic surgery during the covid-19 pandemic: guidelines developed by the international consensus group (ICM). The Journal of Bone and Joint Surgery. 2020; Disponible en: https://www.ncbi.nlm.nih.gov/pubmed/.

7. Giacomelli A, Pezzati L, Conti F, Bernacchia D, Siano M, Oreni L, et al. Self-reported olfactory and taste disorders in SARS-CoV-2 patients: a cross-sectional studylRequest PDF. Clin Infect Dis. 2020; Available in: https://www.ncbi.nlm.nih.gov/pubmed/.

8. $\mathrm{Xu} \mathrm{XW,} \mathrm{Wu} \mathrm{XX,} \mathrm{Jiang} \mathrm{XG,} \mathrm{Xu} \mathrm{KJ,} \mathrm{Ying} \mathrm{LJ,} \mathrm{Ma} \mathrm{CL,} \mathrm{et} \mathrm{al.} \mathrm{Clinical} \mathrm{findings} \mathrm{in} \mathrm{a} \mathrm{group} \mathrm{of} \mathrm{patients} \mathrm{infected}$ with the 2019 novel coronavirus (SARS-Cov-2) outside of Wuhan, China: Retrospective case series. BMJ. 2020; 368: m606. Available in: https://www.ncbi.nlm.nih.gov/pubmed/.

9. Huang C, Wang Y, Li X, Ren L, Zhao J, Hu Y, et al. Clinical features of patients infected with 2019 novel coronavirus in Wuhan, China. Lancet. 2020; 395 (10223): 497-506. Available in: https://www.ncbi.nlm. nih.gov/pubmed/.

10. Chen N, Zhou M, Dong X, Qu J, Gong F, Han Y, et al. Epidemiological and clinical characteristics of 99 cases of 2019 novel coronavirus pneumonia in Wuhan, China: a descriptive study. Lancet. 2020; 395 (10223): 507-513. Available in: https://www.ncbi.nlm.nih.gov/pubmed/.

11. Leung CC, Lam TH, Cheng KK. Mass masking in the COVID-19 epidemic: people need guidance. Lancet. 2020; 395 (10228): 945. Available in: https://www.ncbi.nlm.nih.gov/pubmed/.

12. COVID-19 Guidelines for Triage of Orthopaedic Patients. [Online March 24, 2020] Available in: https:// www.facs.org/covid-19/clinical-guidance/elective-case/orthopaedics.

13. Massey PA, McClary K, Zhang AS, Savoie FH, Barton RS. Orthopaedic surgical selection and inpatient paradigms during the coronavirus COVID-19 pandemic. J Am Acad Orthop Surg. 2020; 28 (11): 436-450.

14. Zhou F, Yu T, Du R, Fan G, Liu Y, Liu Z, et al. Clinical course and risk factors for mortality of adult inpatients with COVID-19 in Wuhan, China: a retrospective cohort study. Lancet. 2020; 395 (10229): 1054-1062. Available in: https://www.ncbi.nlm.nih.gov/pubmed/.

15. Courtney PM, Whitaker CM, Gutsche JT, Hume EL, Lee GC. Predictors of the need for critical care after total joint arthroplasty: An update of our institutional risk stratification model. Journal of Arthroplasty. 2014; 29 (7): 1350-1354. Available in: https://www.ncbi.nlm.nih.gov/pubmed/.

16. Lipira AB, Sood RF, Tatman PD, Davis JI, Morrison SD, Ko JH. Complications Within 30 Days of Hand Surgery: An Analysis of 10,646 Patients. Journal of Hand Surgery. 2015; 40 (9): 1852-1859.e3. Available in: https://www.ncbi.nlm.nih.gov/pubmed/.

17. Safely resuming elective surgery as COVID-19 curve flattens: ACS, ASA, AORN and AHA develop roadmap for readiness I AHA. Available in: https://www.aha.org/press-releases/2020-04-17-safelyresuming-elective-surgery-covid-19-curve-flattens-acs-asa-aorn.

18. Local Resumption of Elective Surgery Guidance. [Accessed April 22, 2020] Available in: https://www. facs.org/covid-19/clinical-guidance/resuming-elective-surgery.

19. Joint Statement: Roadmap for Resuming Elective Surgery after COVID-19 Pandemic. [Accessed April 22, 2020] Available in: https://www.facs.org/covid-19/clinical-guidance/roadmap-elective-surgery.

20. Bhattacharyya T, lorio R, Healy WL. Rate of and risk factors for acute inpatient mortality after orthopaedic surgery. J Bone Joint Surg Am. 2002; 84 (4): 562-572. Available in: https://www.ncbi.nlm.nih.gov/pubmed/.

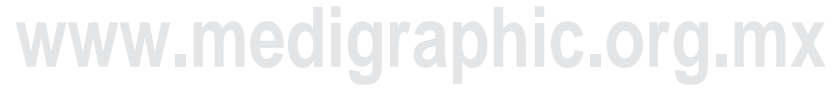

